\title{
Automotive Blind-Zones: A Review of Legislation and the Use of Close-Range Camera Systems
}

\author{
Ciarán Hughes Martin Glavin Edward Jones \\ Connaught Automotive Research Group \\ Department of Electronic Engineering \\ College of Engineering and Informatics \\ National University of Ireland, Galway \\ Nuns Island, Galway, Ireland \\ $+353-91-492605$ \\ \{ciaran.hughes, martin.glavin, edward.jones\}@nuigalway.ie \\ Patrick Denny \\ Vision Research and Systems' Validation Group \\ Valeo Vision Systems \\ I.D.A. Business Park \\ Tuam, Co. Galway, Ireland \\ +353-93-23184 \\ patrick.denny@valeo.com
}

\begin{abstract}
The development of vehicular electronic vision systems for the automotive market is a growing field, driven in particular by customer demand to increase the safety of vehicles both for drivers, and for other road users, including Vulnerable Road Users (VRUs), such as pedestrians. Close-range automotive camera systems are designed to display the areas in the close vicinity of the vehicle to the driver, typically covering the blind-zones of the vehicle. Customer demand is matched by legislative developments in a number of key automotive markets; for example Europe, Japan and the United States are in the process of introducing legislation to aid in the prevention of fatalities to vulnerable road users, with emphasis on the use of vision systems. In this paper we discuss some of the factors that have promoted the introduction of this legislation. We show also that, by the use of wide-angle camera systems, these legislative requirements can be met.
\end{abstract}

\section{Keywords}

automotive camera, wide-angle camera, close-range camera, accident statistics, global legislation, blind-zone, blind-spot, vulnerable road user

\section{INTRODUCTION}

Safety in vehicles is an ever growing concern in most modernised countries. This has led to several initiatives being undertaken in both Europe and North America. For ex-

ISVCS 2008 July 22 - 24, 2008, Dublin, Ireland. Copyright (C)2008 ISBN \# 978-963-9799-27-1 ample, the European New Car Assessment Program (Euro NCAP) [2], which was established in 1997, provides objective information on the safety of drivers and passengers in cars in crash situations. In a study commissioned by the Euro NCAP, $94 \%$ of respondents list safety in vehicles as a major concern [15]. There are similar organisations in both Japan [17] and the United States [4] (known as JNCAP and USNCAP, respectively).

However, more recently, the protection of VRUs, e.g. pedestrians and cyclists, has increased in importance. VRUs hidden in a vehicle's blind-zone ${ }^{1}$ is a major cause of VRU injuries and fatalities globally, as we show later in section 2.1. It is somewhat ironic to note that, with vehicle manufacturers following customer demand by increasing the emphasis on improving the NCAP ratings of their vehicles by strengthening and increasing the size of the vehicles' A-pillars (the vertical or near vertical shaft of material that supports the vehicle roof on either side of the wind-screen), they can also considerably increase the size of the blind-zones caused by those A-pillars.

To match the consumer desire for increased safety in vehicles, the European Union have introduced legislation that requires Large Goods Vehicles (LGVs) ${ }^{2}$ to have large portions of their blind-zones made visible to the driver. Japan is also in the process of introducing similar legislation. While European and Japanese legislation focuses primarily on LGV

\footnotetext{
${ }^{1}$ The term "blind-zone" is used in preference over the other commonly used term "blind-spot", as this is the term used in some of the jurisdictions described in this paper, and more accurately describes the areas that cannot be seen by a driver of a vehicle.

${ }^{2}$ The term "large goods vehicle" is used in this paper, instead of the term "heavy goods vehicle", as this is the terminology used in most European Union documentation due to the fact that the word "heavy" does not have a direct translation in all European languages.
} 
safety, in the United States legislation targeted at privately owned vehicles is proposed with the aim of preventing children being injured while within a moving vehicles blindzone.

Wide-angle/fish-eye lenses can be used by automotive manufacturers to provide information about a vehicle's blindzones to the driver. We will show that, considering the size of certain vehicles' blind-zones, in many cases a camera with a standard field-of-view will be unable to adequately display the blind-zone to the driver. In such cases, wide-angle cameras must be employed. While it is beyond the scope of this paper to investigate the side-effects of using wide-angle cameras, it should be noted that considerable distortion can be introduced when using such devices, although the distortion can be removed via appropriate calibration/correction.

In section 2, we identify the blind-zones that are present in all cars, Sports Utility Vehicles (SUVs) and LGVs. We also examine some statistics for fatalities and injuries on the roads that are linked to vehicle blind-zones in Europe and the United States. In section 3, we examine the legislation from three large automotive markets (Europe, the United States and Japan) that is designed to protect against VRU death and injury due to blind-zones. Finally, in section 4 we suggest how camera systems can be used to meet the requirements of the legislation and display the blind-zones to the driver, particularly by the use of wide-angle camera systems.

\section{VEHICLE BLIND-ZONES}

A vehicle's blind-zones are the areas around the vehicle that cannot be seen directly by the driver by looking forward or by using any of the vehicles' standard rear-view or wing mirrors. Figure 1 shows the areas around a vehicle that are not visible by the driver of a typical vehicle. The sizes of these areas are determined by the size and design of the vehicle and mirrors, and will vary according to car model and manufacturer.

Consumers Union [8] has examined the rearward blind-zone on many private (i.e. non-commercial) vehicles. The zone was measured by determining how far behind the vehicle a 28-inch (0.71 metre) traffic cone representing a young child needed to be before a person, seated in the driver's seat, could see its top while looking through the rear window. For a 5 -foot 8-inch (1.73 metres) tall driver, the distance measured was up to 44 feet (13.41 metres) for a commercially available four-wheel drive Sports Utility Vehicle (SUV) registered in 2006. In the same vehicle, the blind-zone distance for a 5 -foot 1-inch (1.55 metres) driver extends to 69-feet (21.03 metres).

The blind-zone for LGVs is naturally much larger than that of a private vehicle. Ehglen and Paidla [10] calculated the forward blind-zones of a given LGV as shown in figure 2 . Furthermore, the rearward blind-zones of LGVs tend to be very large; we have measured that several LGVs have a rearward blind-zone that can extend up to 65 metres behind the vehicle on the ground plane.

In summary, the presence of vehicle blind-zones has been shown to be a significant factor in VRU injuries and fatali-

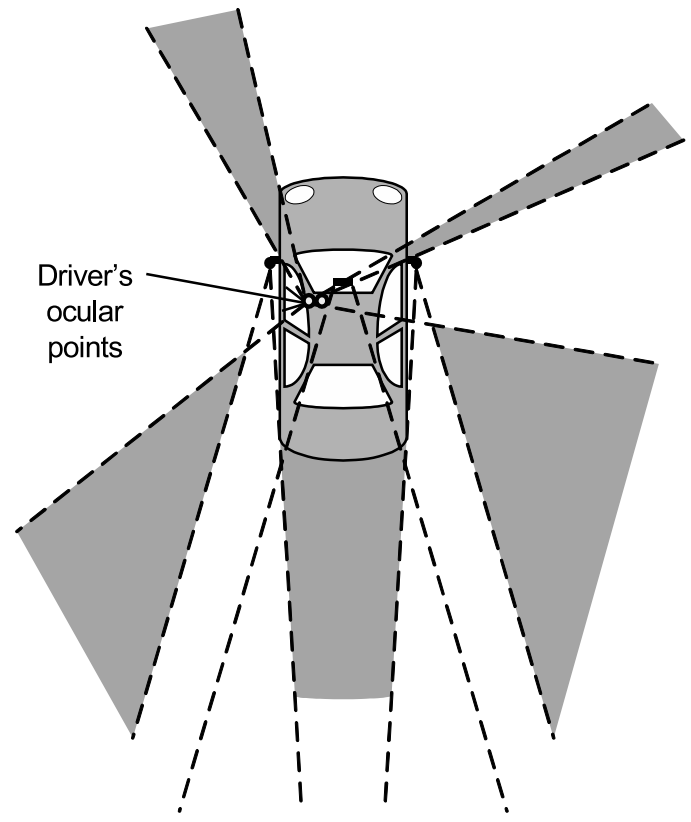

Figure 1: The five blind-zone areas (shown as the shaded area) in a standard left-hand drive car. The sizes of the blind-zones are dependent on the design of the car and the viewing angle of the mirrors.

ties.

\subsection{Blind-zone accident statistics}

\subsubsection{Europe}

Official statistics for VRU deaths due to the victims not being visible to the driver of a vehicle in Europe are unavailable. However, the European Commission's CARE Road Accident Database [11] claims that there were 3,961 VRU pedestrian fatalities within urban areas in 2005. It is reasonable to assume that a significant portion of these deaths were caused by the VRU not being visible to the driver of the vehicle.

This assumption is supported by several statistics; the European Commission Directorate-General for Energy and Transport estimates that the lack of visibility in the blind-zone towards the rear of a vehicle directly causes 500 deaths a year in the EU [12]; The Commission of the European Communities estimates that every year, approximately 400 European road users lose their lives in accidents with LGVs, because the driver did not see them, when turning right [6]; the UK Health and Safety Executive reports that in 12 months between 2005 and 2006, 18 people were killed and 620 sustained major injuries due to the intentional motion of the vehicle (either forwards or backwards) in the workplace [22]; the Organisation for Economic Co-operation and Development (OECD) reports that $60 \%$ of child casualties occur within 500 metres of their home [20].

\subsubsection{United States}

Statistics for the United States are equally disjointed, with no official statistics directly available for VRU injuries due to vehicle blind-zones. However, the Kids and Cars Organi- 


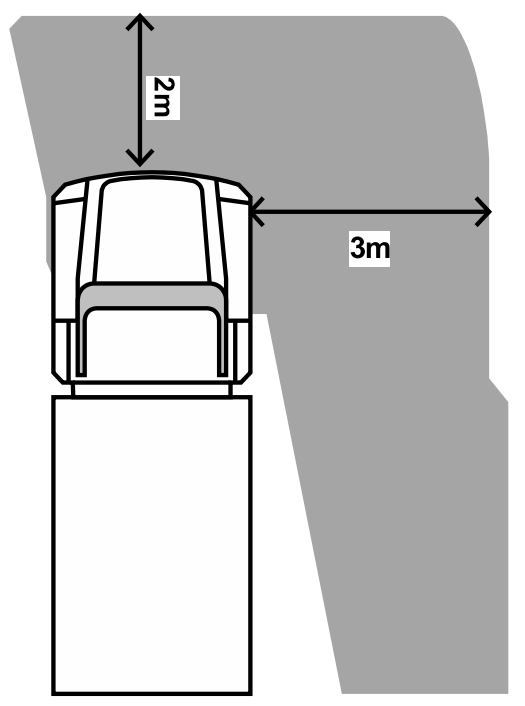

Figure 2: Blind-zones (shown as the shaded area) around the front of a given left-hand drive LGV.

sation in the United States [3] claims that between 2002 and 2006 there have been 941 children killed in non-traffic accidents in the United States. They further claim that $49.5 \%$ (or 466 children) of the fatalities were due to the vehicle reversing while children were present in a vehicle's rearward blind-zone.

The Centers for Disease Control and Prevention (CDC) [18] in a study between July 2000 and June 2001, reported that there were an estimated 9,160 non-fatal injuries to children in non-traffic automotive accidents, with approximately $20 \%$ (or 1,832 children) of these injuries caused by the vehicle moving backwards. Between 2001 and 2003, the CDC reported that an estimated 7,475 children (2,492 per year) were treated for moving vehicle backover injuries [19]. Again, while the blind-zone is not directly implicated in these injuries, it is reasonable to assume that a significant proportion of these injuries were due to the children being present in the vehicle's blind-zone.

Wang and Knipling [25] estimated that lane change/merge crashes in 1991 accounted for approximately 244,000 policereported crashes with 224 associated fatalities. Furthermore, the authors reported that the principal causal factor in such crashes is that the driver "did not see other vehicle".

\section{LEGISLATION PROMOTING THE USE OF VEHICULAR CAMERA SYSTEMS}

Due to the increasing awareness of the vulnerability of pedestrians, legislation has been introduced, or is in the process of being introduced, in several jurisdictions around the world. In this paper, we describe the legislative requirements or potential requirements for the EU, Japan and the United States.

\subsection{Europe}

In the EU, legislation in the form of Directive 2003/97/EC [13] has already been introduced. Although the initial re-

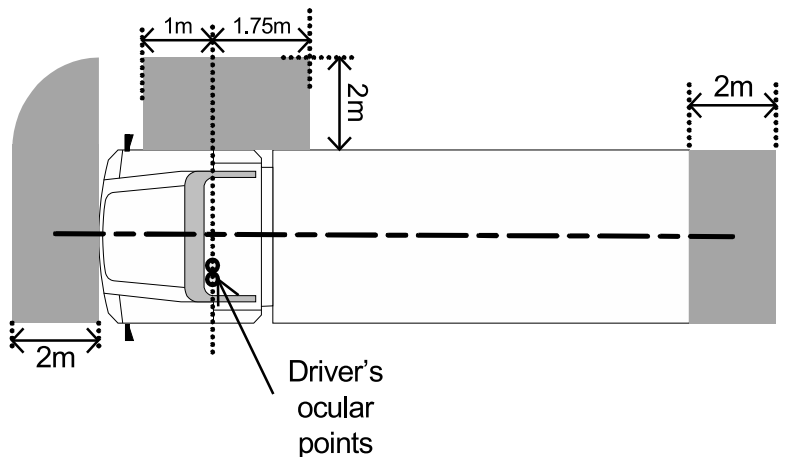

Figure 3: Area required by Directive 2003/97/EC to be visible to drivers of (left-hand drive) LGVs via the use of indirect vision systems (not including areas covered by the standard and wide-angle wingmirrors).

quirements of this directive help reduce accidents caused by the blind-zones of LGVs and improve road safety for new vehicles circulating from 2006/2007 onwards, the legislation did not cover the existing fleet of lorries in EU. Partially due to the fact that it was estimated that existing fleets will not be fully replaced until 2023, Directive 2007/38/EC [14] was introduced requiring the retrofitting of the required indirect vision systems to all existing fleets. When these directives are fully applied to all LGVs, including new and existing fleets, the European Parliament estimates that approximately 1200 road fatalities could be prevented, which translates into around $€ 2.4$ billion to the EU member states, in terms of societal costs [7].

The shaded areas in figure 3 shows the areas of a left-hand drive LGV's ${ }^{3}$ environment that must be visible to the driver via the use of "Indirect Vision Systems (IVS)", as required by these directives. Examples of IVS's include mirrors in addition to the standard rear-view and wing-mirrors, as well as camera-monitor devices. However, practical problems arise with the use of additional mirrors as the extra mirrors themselves can introduce additional blind-zones, as well having additional cost and styling implications.

There is a clear provision for the use of camera-monitor devices for the coverage of vehicle blind-zones in this directive. In fact, the use of camera-monitor devices over mirrors is often not only desirable, but necessary. For example, it is practically impossible to cover the area at the rear of an LGV with mirrors alone, so camera-monitor systems are the only practical solution.

\subsection{Japan}

In Japan, there is also proposed legislation to enable objects within a portion of a medium and large vehicles blind-zones to be detected by the driver, either directly, by mirror or by camera-monitor device (See figures 4 and 5) [24]. For the purpose of the proposed legislation, the driver of the LGV must be able to notice a cylinder 1 metre high with

${ }^{3}$ While the figure shows a left-hand drive vehicle, for righthand drive vehicles within the jurisdiction of the legislation, the areas of required coverage are reversed 


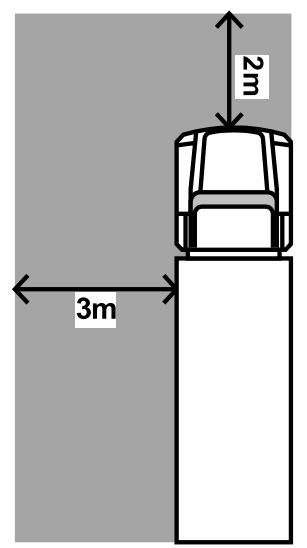

Figure 4: Proposed Japanese legislation: area in which a cylinder ( $1 \mathrm{~m}$ high, $0.3 \mathrm{~m}$ diameter) must be at least partially visible to the driver of a right-hand drive LGV (greater than 7.5 tonnes).

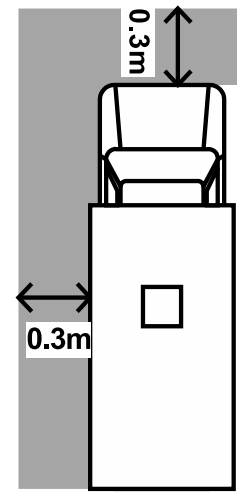

Figure 5: Proposed Japanese legislation: area in which a cylinder ( $1 \mathrm{~m}$ high, $0.3 \mathrm{~m}$ diameter) must be at least partially visible to the driver of a right-hand drive LGV (less than 7.5 tonnes).

a diameter of 0.3 metres placed anywhere within the coverage areas. However, in this legislation, it is proposed that objects within blind-zones caused by A-pillars and external mirrors need not be visible to the driver of the vehicle [23].

\subsection{United States}

In the United States, proposed legislation, in the form of S.694 (The Cameron Gulbransen Kids and Cars Safety Act of 2007) [1], is designed to protect against children being injured or killed in non-traffic incidents, such as when the vehicle is reversing. Specifically relating to the possible use of cameras, the S.694 bill requires a rearward visibility that enables the driver to detect the presence of a person in the rear blind-zone of a vehicle in order to prevent injury or death.

\section{AUTOMOTIVE CAMERA SYSTEMS}

As shown in figure 6, standard lens camera systems (e.g. $45^{\circ}$ Field-Of-View (FOV) lenses) are unable to fully cover the blind-zone of some SUVs. Considering that camera-monitor systems generally display a "reference" point (i.e. part of

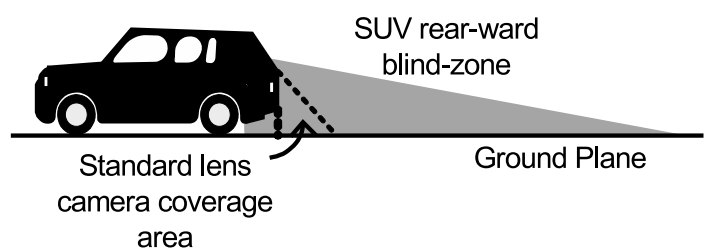

Figure 6: Example coverage area for standard lens camera (e.g. 45 degree field-of-view).

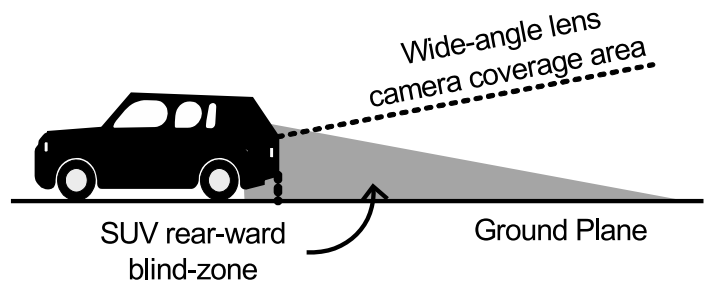

Figure 7: Example coverage area for wide-angle lens camera (e.g. 100 degree field-of-view).

the body of the vehicle) on screen, a standard lens camera with FOV of $45^{\circ}$ can only cover perhaps $1 \mathrm{~m}$ of the SUV blind-zone. Figure 7 shows how the use of a wide-angle lens camera system (e.g. $>100^{\circ}$ Field-Of-View (FOV) lenses) enables the entire SUV rear-ward blind-zone to be covered.

Figure 8 shows sample areas that could potentially be displayed to a driver if a number standard VGA cameras with wide-angle lens systems were mounted on an LGV. Camera 1 is a $135^{\circ}$ wide-angle camera, located approximately halfway down the length of the LGV and $3 \mathrm{~m}$ off the ground plane. The optical axis of camera 1 is tilted at $15^{\circ}$ from the side of the LGV trailer. Camera 2 is a $135^{\circ}$ wide-angle camera, located in the middle of the front cabin and $2 \mathrm{~m}$ off the ground plane. The optical axis of camera 2 is tilted at $20^{\circ}$ from the front face of the cabin.

Figure 8 shows the areas in the vicinity of the vehicle that can be displayed to the driver (if video from both cameras were corrected for distortion that is evident in wideangle cameras, using one of several calibration/correction techniques described in $[21,9,5,16]$, or other literature). From this figure, it can be seen that such a camera system would cover all the blind-zones of the LGV shown in figure 2, and would meet the requirements of both the EU Directive 2003/97/EC (figure 3) and the proposed Japanese legislation (figures 4 and 5 ).

The coverage of these distortion corrected wide-angle cameras extend far beyond that required by the legislations mentioned in the directions perpendicular to the vehicle side/front. However, these wide fields-of-view are necessary to obtain the coverage along the length and across the front of the vehicle.

The rearward coverage area required by the EU Directive (figure 3) could be covered by a standard lens camera. However, a potentially better product would be a wide-angle 


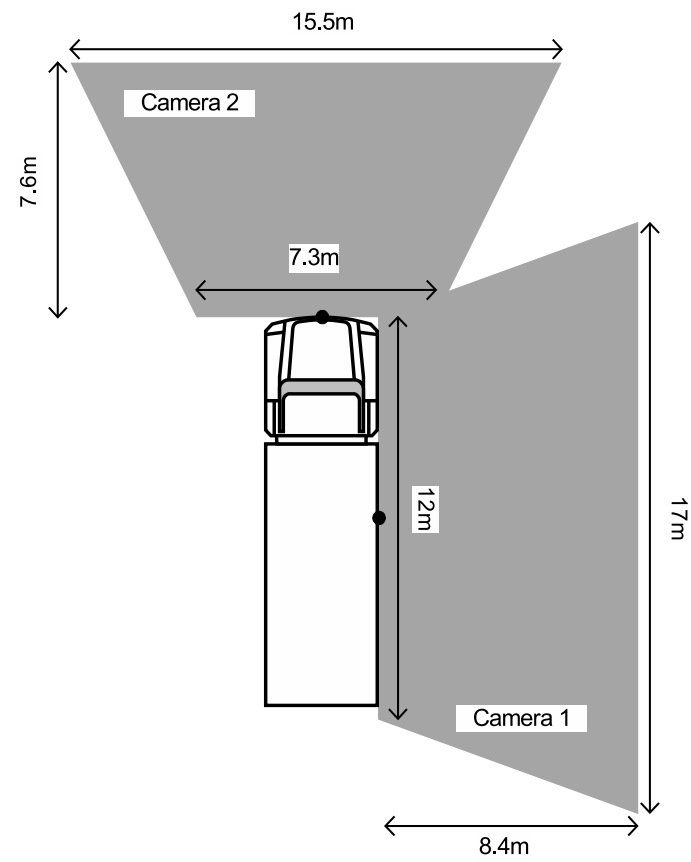

Figure 8: Example of a potential camera system for LGVs that would meet the requirements of the EU Directive 2003/97/EC and the proposed Japanese legislation.

camera mounted appropriately to display more of the rearward blind-zone to the driver. As we have already described in section 2, the rearward blind-zone of certain LGVs can be up to $65 \mathrm{~m}$, which is significantly larger than the area requiring coverage under the directive.

\section{CONCLUSIONS}

In this paper, we have discussed the rationale within the automotive industry for the on-vehicle use of camera systems. Specifically, with increasing numbers of vehicles on the world's roads, statistics show that a significant percentage of fatalities on the road are caused by VRUs being hidden in a vehicles blind-zones, and that increased visibility of these blind-zones will result in a reduced number of fatalities.

While customer demand for products that give information about a vehicles blind-zone to the driver is already high, pending and existing legislation is, de facto, making the placement of such cameras on vehicles a necessity, particularly for large SUVs and LGVs. However, while small vehicles are exempt from the European and Japanese legislation, in the United States legislation specifically targets the smaller private vehicles.

Ultimately, camera systems are becoming a necessity to increase vehicle safety, particularly in SUVs and LGVs. More specifically, we have shown that wide-angle camera systems would be required to display the potentially very large areas of coverage required by the legislation discussed. Indeed, we have shown in figure 8 that the use of a minimal number of wide-angle camera systems can be used to display the blindzones to the driver of the vehicle far beyond what is required by legislation.

\section{ACKNOWLEDGEMENTS}

This research is funded by Enterprise Ireland and Valeo Vision Systems (formerly Connaught Electronics Ltd.) under the Enterprise Ireland Innovation Partnerships Scheme.

\section{REFERENCES}

[1] American Library of Congress, S.694: Cameron Gulbransen Kids and Cars Safety Act of 2007, http://thomas.loc.gov/cgi-bin/query/z?c110:S.694:, accessed March 2008.

[2] European New Car Assessment Program (Euro NCAP) website, http://www.euroncap.com, accessed March 2008.

[3] Kids and Cars Organisation website, http://kidsandcars.org/, accessed March 2008.

[4] US National Highway Traffic Safety Administration (USNCAP), http://www.safercar.gov/, accessed March 2008.

[5] A. Basu and S. Licardie. Alternative models for fish-eye lenses. Elsevier Pattern Recognition Letters, 16(4):433-441, November 1995.

[6] Commission of the European Communities. Commission Staff Working Document: Accompanying document to the proposal for a directive of the European Parliament and of the Council on the retrofitting of mirrors to heavy goods vehicles registered in the Community Full Impact Assessment $\operatorname{COM}(2006) 570$, October 2006.

[7] Commission of the European Communities. Impact Assessment Factsheet FII/2006/0183 for procedure: Road safety-Retrofitting of mirrors to existing fleet of heavy goods vehicles COD/2006/0183, November 2006.

[8] Consumers Union. Consumer Reports: Blind-zone measurements, http://www.consumerreports.org/, accessed March 2008.

[9] F. Devernay and O. Faugeras. Automatic calibration and removal of distortion from scenes of structured environments. In Proceedings of the SPIE Investigative and Trial Image Processing Conference, volume 2567, pages 62-72, September 1995.

[10] T. Ehlgen and T. Paidla. Maneuvering aid for large vehicle using omnidirectional cameras. In IEEE Workshop on Applications of Computer Vision, pages 17-17, Feb. 2007.

[11] European Commission. Community Database on Accidents on the Roads in Europe (CARE) website, http://ec.europa.eu/, accessed March 2008.

[12] European Commission Directorate-General for Energy and Transport. Halving the number of road accident victims in the EU by 2010: A shared responsibility. European Road Safety Action Programme, October 2004.

[13] European Parliament and Council. Directive 2003/97/EC of 10 November 2003 on the approximation of the laws of the Member States relating to the type-approval of devices for indirect vision and of vehicles equipped with these devices, 
amending Directive 70/156/EEC and repealing Directive $71 / 127 /$ EEC.

[14] European Parliament and Council. Directive $2007 / 38 /$ EC of 11 July 2007 on the retrofitting of mirrors to heavy goods vehicles registered in the Community.

[15] A. Hobbs. Euro NCAP/MORI survey on consumer buying interests (speech and presentation). In Proceedings of the Euro NCAP Conference: Creating a Market for Safety - 10 years of Euro NCAP, 2005.

[16] C. Ishii, Y. Sudo, and H. Hashimoto. An image conversion algorithm from fish eye image to perspective image for human eyes. In Proceedings of the IEEE/ASME International Conference on Advanced Intelligent Mechatronics, volume 2, pages 1009-1014, July 2003.

[17] Japanese National Agency for Automotive Safety and Victims Aid. New Car Assessment (JNCAP), http://www.nasva.go.jp/mamoru/indexe.html, accessed March 2008.

[18] E. McLoughlin, J. Middlebrooks, J. Annest, P. Holmgreen, and A. Dellinger. Injuries and Deaths Among Children Left Unattended in or Around Motor Vehicles - United States, July 2000-June 2001. Centers for Disease Control and Prevention (United States Department of Health and Human Services) Morbidity and Mortality Weekly Report, 51(26):570-572, July 2002.

[19] R. Patel, A. Dellinger, and J. Annest. Nonfatal motor-vehicle-related backover injuries among children - United States, 2001-2003. Centers for Disease Control and Prevention (United States Department of Health and Human Services) Morbidity and Mortality Weekly Report, 54(6):144-146, February 2005.

[20] Scientific Expert Group on the Safety of Vulnerable Road Users. DSTI/DOT/RTR/RS7(98)1/FINAL Safety of Vulnerable Road Users. Technical report, Organisation for Economic Co-operation and Development (OECD), August 1998.

[21] R. Tsai. A versatile camera calibration technique for high-accuracy 3D machine vision metrology using off-the-shelf TV cameras and lenses. IEEE Journal of Robotics and Automation, 3(4):323-344, August 1987.

[22] UK Health and Safety Executive. Statistics: Number of workplace transport injuries, http://www.hse.gov.uk/workplacetransport/, accessed March 2008.

[23] UNECE Working Party on General Safety Provisions (GRSG). GRSG-83-Inf15e: Outline of Draft

Amendment to ECE Regulation No.46 (Draft Requirements for Driver's Field of Vision of Immediate Frontward and Sideward), October 2002.

[24] UNECE Working Party on General Safety Provisions (GRSG). GRSG-89-26: Proposal for Step-2 revision of Regulation No. 46, October 2005.

[25] J. Wang and R. Knipling. Lane change/merge crashes: problem size assessment and statistical description. Report No. DOT HS 808-075. Technical report, United States Department of Transportation Publication, 1994. 